\title{
Optical Characterisation of a Fractal Solar Concentrator
}

\author{
Ilia Katardjiev \\ Department of Electrical Engineering, Uppsala University \\ ilia.katardjiev@angstrom.uu.se
}

9 January 2021

\section{Abstract}

An optical characterisation of a fractal light concentrator is presented. The concentrator has a geometrical concentration coefficient of $3 X$ and an acceptance angle of around 60 degrees. Visual observations of the rejected intensity under various angles confirm the theoretically predicted performance.

\section{Introduction}

Fractal concentrators have been recently proposed[1] for light concentration. They comprise an array of elongated optical elements (OE) of general conical shape separated by small gaps of a lower refractive index optical material (typically air) and enclosed at the periphery by a reflecting mirror structure as illustrated in 2D in Figure 1.

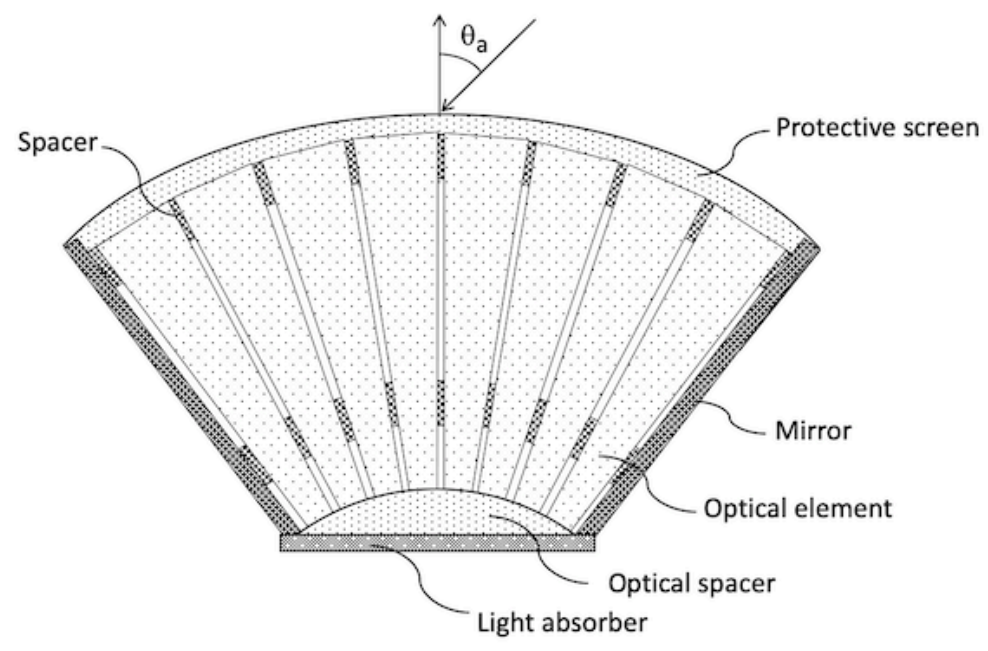

Figure 1. Schematic illustration of a fractal concentrator.

The OE's geometry can vary depending on the specific design. In this case, the OEs represent truncated triangular pyramids made of PMMA. It is shown[1] that in this type of arrangement each OE has an acceptance angle $\theta_{a}$ with respect to the OE's axis of symmetry is identical to that of conical waveguides with ideally reflecting walls which is given by

$\theta_{a}=\operatorname{asin}\left[\frac{n_{1}}{n_{2} \sqrt{C_{g}}}\right]-\varepsilon$

where $n_{1}$ is the refractive index of the OEs, $n_{2}$ is the refractive index of the optical material surrounding the OEs, $\varepsilon$ is an angle proportional to the solid angle occupied by an $O E$ and $C_{g}$ is 
the geometrical concentration. Due to the elongated geometry of the $\mathrm{OE} \varepsilon$ is much smaller than the first term in eq. (1), particularly at low $C_{g}$. Hence,

$\theta_{a} \approx \operatorname{asin}\left[\frac{n_{1}}{n_{2} \sqrt{C_{g}}}\right]$

The acceptance angle of the concentrator itself with respect to the vertical z-axis is dependent on the angular distribution of the incident light flux due to the annular shape of its acceptance surface. Thus, for a totally diffuse light source the concentrator acceptance angle is equal to that given by eq. (1). For a point light source, however, the concentrator acceptance angle is also dependent on the solid angle occupied by the concentrator itself and is given by

$\theta_{a} \approx \operatorname{asin}\left[\frac{n_{1}}{n_{2} \sqrt{C_{g}}}\right]-\frac{\alpha}{2}$

where $\alpha$ is the angular aperture of the conical mirror surface. Hence, in the case of point light sources it is advantageous to design concentrators with a minimal aperture $\alpha$, i.e. a minimal number of OEs. The smallest possible number in the case of triangular OEs is six. The objective of this communication is to present initial optical characterisation of a fractal concentrator at low concentration levels in view of diffuse light sources.

\section{Design of the optical elements}

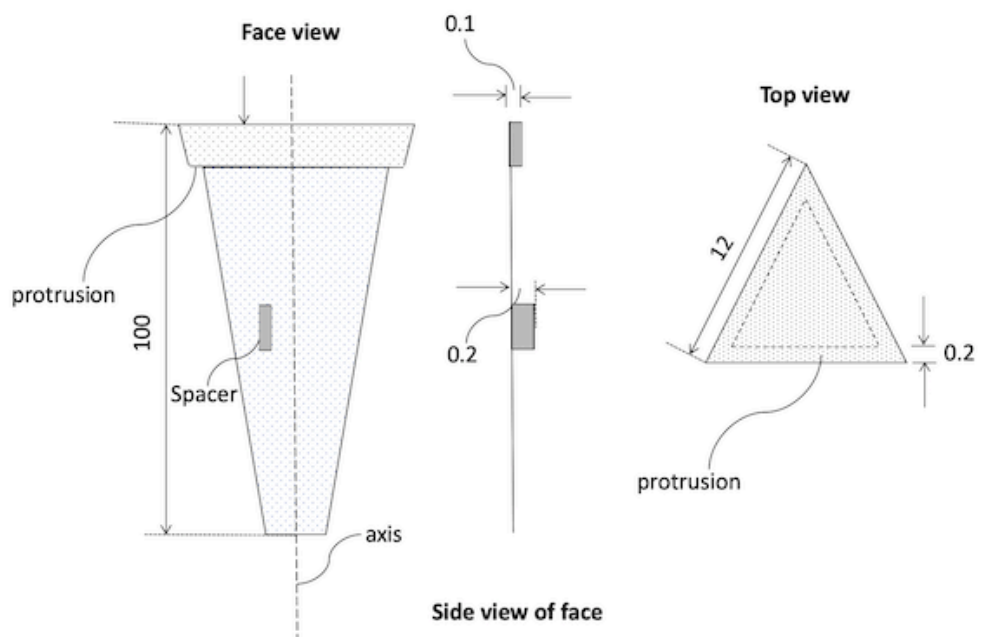

Figure 2. Design of the optical elements

The specific design follows the design rules described in reference 1 and is illustrated in Figure 2 where all dimensions are in millimetres. Specifically, the OEs represent truncated triangular pyramids of height $100 \mathrm{~mm}$. The side of the front surface is $12 \mathrm{~mm}$ while the bottom side is $6,9 \mathrm{~mm}$ resulting in a concentration $3 X$. The air gaps between the OEs are defined by two sets of spacers. The first one represents $0.1 \mathrm{~mm}$ thick protrusions around the periphery of the top surface while the second one represents spacers positioned at some distance from the top surface having a height twice that of the protrusion. Note the asymmetric positioning of the spacer relative to the axis of the sidewall. This arrangement results in the automatic formation of a $0.2 \mathrm{~mm}$ gap when two neighbouring faces are 
brought into contact. The use of two sets of spacers is superfluous since only one set is sufficient for the definition of a gap between two planar surfaces. The gap width need not be uniform either. Decreasing the gap widths at the top surface reduces rejection while decreasing the gap widths at the exit increases $C_{g}$.

Finally, the number of OEs in the concentrator is chosen to be 150 resulting in an acceptance aperture representing a hexagon with a side of approximately $60 \mathrm{~mm}$.

\section{Experimental}

The OEs were fabricated by injection moulding of PMMA. The peripheral mirror structure represents a truncated hexagonal pyramid where the reflecting surfaces point to the inside as illustrated in Figure 3.

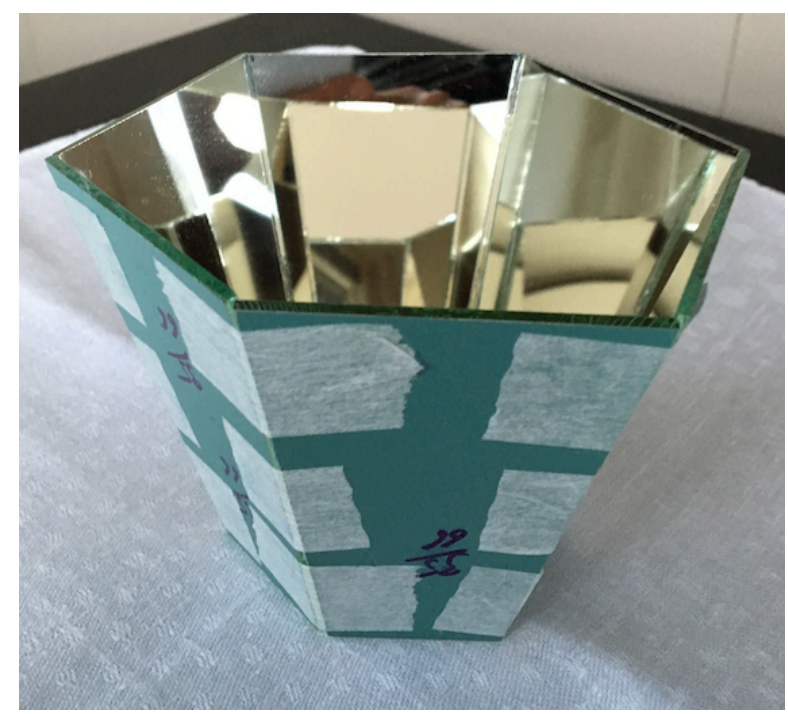

Figure 3. Hexagonal mirror structure

A number of identical concentrators were subsequently assembled. Figure 4 shows two concentrators side by side under identical illumination conditions. The one on the left has a diffusely reflecting absorber and consequently reflects (rejects) most of incident light back to the ambient apart from obvious absorption losses. The transmittance of the concentrator is calculated[1] to be around $84 \%$ with an acceptance angle of 60 degrees. The concentrator on the right has a black absorber meaning that it absorbs most of the light arriving on its surface. Hence, the only light emanated from the concentrator is the rejected fraction by the concentrator itself. The black colour of the surface indicates that the concentrator does not detectably emit light in the normal direction apart from the gap rejections. 


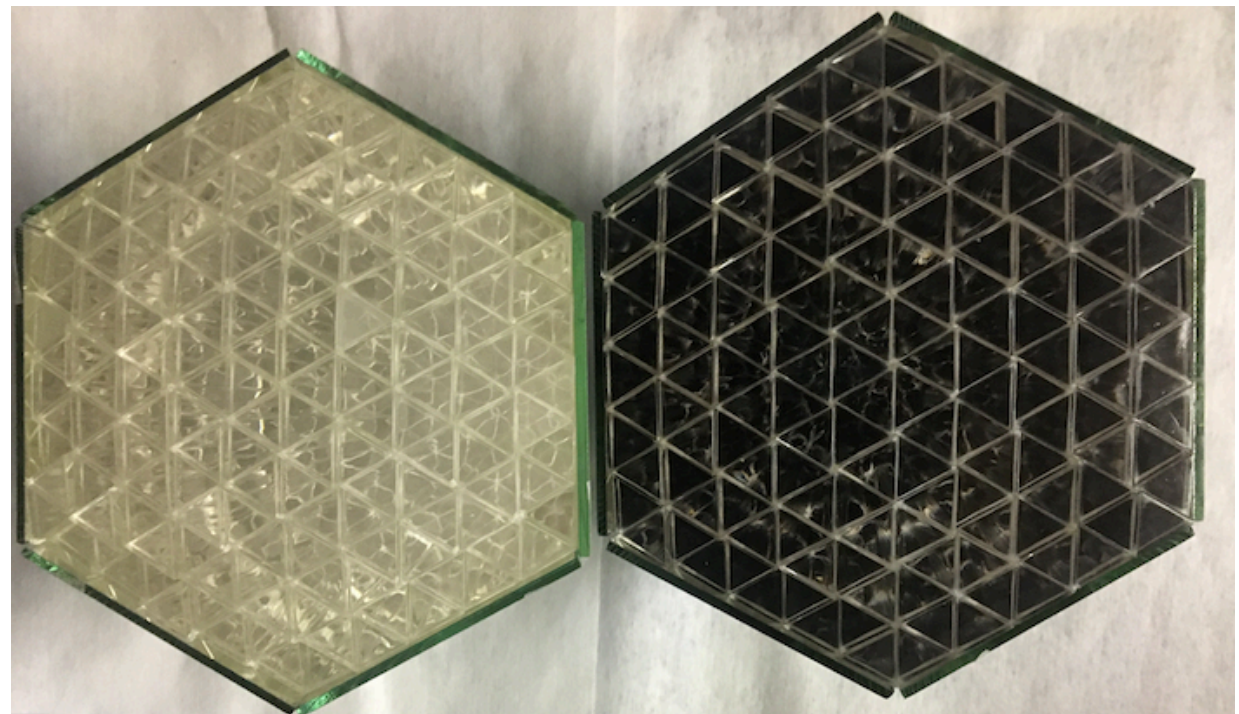

Figure 4. Top view of two identical concentrators but with different absorbers

Observation of the light emission from the concentrator in any direction within the acceptance angle gave the same result as illustrated in Figure 5.

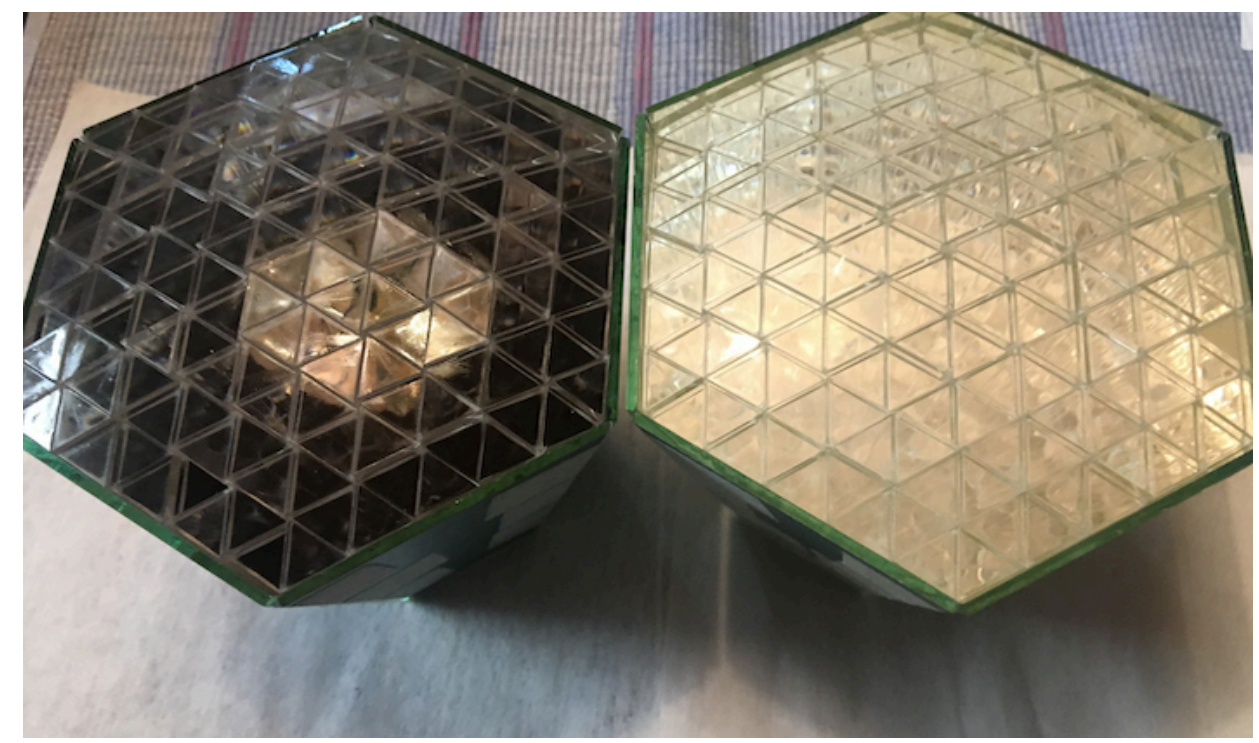

Figure 5. Angled view of the concentrators in Figure 4.

It represents a photograph of the two concentrators viewed simultaneously at an angle of about 45 degrees. All bright features on the black surface represent obvious reflections off the top surface and most notably an image of the illumination source. Visual observations at angles outside the acceptance angle show a feeble greyish colour since the amount of light entering the concentrator at these angles is rather small as its intensity scales with the cosine of the angle of incidence.

\section{Conclusions}

The optical observations evidence that the concentrator performs according to the theoretical predictions[1]. Precise quantitative measurements are under way. 


\section{References}

1. I. Katardjiev, A fractal concentrator of direct and indirect sunlight, Clean Energy, https://doi.org/10.1093/ce/zkaa017

\section{Acknowledgements}

The financial support of Vinnova and UUInnovation AB is greatly acknowledged.

\section{Note:}

This is an Open Access article distributed under the terms of the Creative Commons

Attribution Non-Commercial License (http://creativecommons.org/licenses/by-nc/4.0/) 